\title{
Cepheids in Binary and Multiple Systems
}

\author{
László Szabados \\ Konkoly Observatory, P.O.Box 67, H-1525 Budapest XII, Hungary
}

\begin{abstract}
Compilation of the list of Cepheids which are members in binary and multiple systems is in progress. During this project SB-nature of a number of Cepheids was revealed. The frequency of binaries among Cepheids exceeds 60 per cent.
\end{abstract}

Pulsating stars belonging to binary (multiple) systems offer special opportunity for determining the physical parameters of the components (e.g., Evans, 1994). Cepheids are particularly important because of their rôle in calibrating the cosmic distance scale. In view of their importance, the list of Cepheids with companion(s) is being compiled. The list contains some basic parameters for the Cepheids and their companion(s) as well as key references. The table supplemented with additional remarks will be published in the near future.

The use of novel and more accurate observational techniques (e.g., IUEstudy, extensive radial velocity measurements) has resulted in discovering companions to an unexpectedly large percentage of Cepheids. However, the earlier observational material is not obsolete. It is worth mentioning that Joy's (1937) classical radial velocity study is still invaluable because the comparison of the modern data with Joy's early (therefore obviously less accurate) measurements often helps revealing variability in the $\gamma$-velocity. Such recent discoveries include SB-nature of Cepheids TZ Mon, CR Ori and YZ Sgr.

Another fact to be emphasised is that Joy's data were collected during a decade-long-interval (or even longer), and the effect of the orbital motion appears as an extra scatter in the radial velocity curves. RU Sct may be a member in a spectroscopic binary system based on this criterion.

The orbital effect has been discovered in other cases, as well. VZ CMa, previously known as having a blue companion (Stobie \& Balona, 1979; Szabados, 1993), has a variable $\gamma$-velocity. Moreover, the comparison of two recent observational series on SV Vul (Gorynya et al., 1992; Bersier et al., 1994) shows that this long-period Cepheid also belongs to an SB-system. The detailed discussion on these (and some more) recently discovered SB-Cepheids is left for a future paper (Szabados, 1995).

Another useful criterion for pointing out companion(s) to Cepheids is the relatively large radial velocity amplitude with respect to the amplitude of the light variation (the AR-method - Szabados, 1993). Based on this parameter, UZ Cas and AK Cep are not solitary Cepheids, either.

Although there are well over a hundred items in the list of Cepheids belonging to binary and multiple systems, a big number of companions have not been discovered yet, as is seen in Figure 1. This histogram shows the number of classical Cepheids as the function of the mean V-magnitude (divided into 


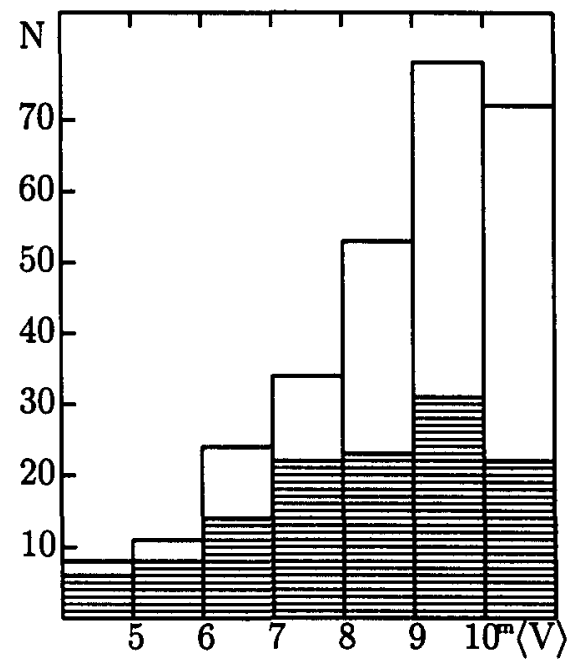

Figure 1. Fraction of the known binaries among Cepheids. The number is plotted against the mean $\mathrm{V}$ brightness for one magnitude wide intervals. Hatched area: known binaries; white rectangles: rest of the sample. Note the high occurrence of binaries among the brightest Cepheids.

one-magnitude wide intervals). The hatched area represents Cepheids known to have companions in each bin. It is clearly visible that the occurrence of binaries exceeds $60 \%$ among the brighter Cepheids. The decreasing frequency of occurrence towards the fainter stars is the result of an obvious selection effect. The observed frequency of binaries among Cepheids of 10th visual magnitude is still about $40 \%$, a larger value than used to be assumed. Nevertheless, dozens of new binaries remain to be discovered among Cepheids brighter than 11th magnitude. For modern instrumentation these stars are not faint any more.

Acknowledgments. Financial assistance from the OTKA T7438 and the Hungarian Academy of Sciences is gratefully acknowledged.

\section{References}

Bersier, D., Burki, G., Mayor, M., \& Duquennoy, A. 1994, A\&AS, 108, 25

Evans, N. R. 1994, ApJ, 436, 273

Gorynya, N. A., Irsmambetova, T. R., Rastorgouev, A.S., \& Samus, N. N. 1992, Pis'ma v Astr. Zhu., 18, No. 9, 777

Joy, A. H. 1937, ApJ, 86, 363

Stobie, R. S., \& Balona, L. A. 1979, MNRAS, 189, 641.

Szabados, L. 1993, in "New Perspectives on Stellar Pulsation and Pulsating Variable Stars", Proc. IAU Coll. No. 139; eds.: J.M. Nemec \& J.M. Matthews, Cambridge Univ. Press, p. 406

Szabados, L. 1995, to be published 